\title{
Incidence and Predictors of Cannabis-Related Poisoning and Mental and Behavioral Disorders Among Patients with Medical Cannabis Authorization: A Cohort Study
}

Arsene Zongo ( $\nabla$ arsene.zongo@pha.ulaval.ca )

Université Laval

Cerina Lee

University of Alberta

Jason R. B. Dyck

University of Alberta

Jihane El-Mourad

Université Laval

Elaine Hyshka

University of Alberta

John G. Hanlon

St. Michael's Hospital, University of Toronto

Dean Eurich

University of Alberta

\section{Research Article}

Keywords: medical cannabis, cannabis use disorder (CUD), cohort study, epidemiology, mental health, behavioral disorder

Posted Date: September 3rd, 2021

DOl: https://doi.org/10.21203/rs.3.rs-753742/v1

License: (c) (1) This work is licensed under a Creative Commons Attribution 4.0 International License. Read Full License 


\section{Abstract}

Objective: As medical cannabis use increases in North America, establishing the safety profile of cannabis is a priority. The objective of this study was to assess rates of emergency department (ED) visits and hospitalizations due to poisoning by cannabis and cannabis-related mental health disorders among medically authorized cannabis patients in Ontario, Canada, between 2014 and 2017.

Methods: This is a retrospective longitudinal study conducted among a cohort of patients who received an authorization to use cannabis for treating various health conditions in Ontario, Canada. The cannabis cohort was selected using data collected in participating Canadian cannabis clinics. Outcomes included: ED visit/hospitalization with a main diagnosis code for cannabis/cannabinoid poisoning; and ED visit/hospitalization with a main diagnosis code for mental/behavioural disorders due to cannabis use. The Spearman correlation and univariate Cox proportional hazard regression was utilized.

Results: From 29153 patients who received medical authorization, 23091 satisfied the inclusion criteria. During a median follow-up of 240 days, 14 patients visited the ED or were hospitalized for cannabis poisoning - with an incidence rate of 8.06 per 10,000 person-years patients ( $95 \% \mathrm{Cl}$ : $4.8-13.6)$. A total of 26 patients visited the ED or were hospitalized for mental and behavioural disorders due to cannabis usewith an incidence rate of 15.0 per 10,000 person-years $(95 \% \mathrm{Cl}$ : $10.2-22.0)$. Predictors of cannabis-related mental and behavioural disorders include prior substance use disorders (drugs and alcohol), other mental disorders, age, diabetes, and chronic obstructive pulmonary disease.

Conclusions: The results suggest that the incidence of cannabis poisoning or cannabis-related mental and behavioural disorders was low among patients who were authorized to use cannabis to treat a health condition. Identified predictors can help to target patients with potential risk of the studied outcomes.

\section{Background}

The increasing medical use of cannabis and cannabis-based products raises safety concerns for patients and public health $(1,2)$. Potential increases in the prevalence of cannabis intoxication, cannabis withdrawal symptoms and cannabis use disorders, are safety issues raised in discussions about potential therapeutic benefits of cannabis for medical use (3). While cannabis intoxication and withdrawal symptoms are acute effects, cannabis use disorder (CUD) is defined as a longer-term mental health condition resulting from the chronic use of cannabis (4-6).

Cannabis poisoning was responsible for 16,884 admissions to emergency departments (EDs) in the United States in 2016, representing $0.014 \%$ of total ED visits for individuals ages 12 and older(7). Recent data collected from the Alberta National Ambulatory Care Reporting System in Canada(8), showed that cannabis-related ED visits and cannabis-related calls to poison control centres increased, after the legalization of non-medical cannabis use (2018). A US study using a nationwide inpatient sample showed that the prevalence of hospitalizations for cannabis abuse and dependence increased from 2002 $(0.52 \%)$ to $2011(1.34 \%)(9)$.In one of the rare study that included only medical cannabis users and 
assessed problematic cannabis use during a 12-month follow-up $(n=265)$, cannabis misuse (for example taking more cannabis than prescribed) and addiction behavior were observed among $26 \%$ and $9 \%$, respectively (10)..

Different factors may predispose some individuals to cannabis poisoning and use disorders (9-11). These include psychiatric comorbidities $(12,13)$, concomitant use of other substances such as heroin, benzodiazepines and cocaine (5), prior substance use disorders $(10,14)$. A study showed that individuals who sought emergency medical care for cannabis poisoning (no distinction between medical and recreational cannabis users) were more likely to be young, male, uninsured, experience economic hardship, reside in urban centers, and experience mental health disorders as compared to individuals admitted for other causes (15).

However, little is known about factors that predict cannabis-induced intoxication and CUD amongst patients who use cannabis for medical purposes, i.e., those who are legally authorized by their care providers to use cannabis to treat specific health conditions. Therefore, this study aimed to assess the incidence rates of ED visits or hospitalization due to 1) poisoning by cannabis and 2) mental and behavioural disorders due to use of cannabis among patients who were medically authorized to use cannabis in Ontario, Canada, between 2014 and 2017. Secondly, we assessed factors that are associated with these outcomes.

\section{Methods}

\section{Study design}

This is a retrospective longitudinal study conducted among a cohort of patients who received an authorization to use cannabis for treating different health conditions in Ontario, Canada. Patients' followup started from their specific date of cannabis authorization.

\section{Study Population}

This study included all patients aged $\geq 18$ years authorized to access medical cannabis from a group of cannabis clinics in Ontario, Canada between April 24, 2014 and March 31, 2017. The cohort has been described in previous papers $(16,17)$. Briefly, in the study period (2014-2017), patients seeking cannabis for medical use in Canada were required to obtain physician approval and administrative authorization from Health Canada. Thus, all patients in our cannabis cohort were legally allowed to use cannabis for medical purposes. These patients were referred to the cannabis clinics by other physicians or selfreferred. Patients who were not eligible for universal health insurance via the Ontario Health Insurance Plan (provided to all people meeting Ontario residency requirements) and those with invalid or duplicate identifiers were excluded. 


\section{Data Source}

The cannabis cohort was selected using data collected in the cannabis clinics. Clinic data was linked to Ontario administrative health data to assess the study outcomes and potential predictors. As described previously, The Institute for Clinical Evaluative Sciences (ICES) provided the health administrative data. These data include individual data files for each beneficiary, inpatient records, physician billings (inpatient and outpatient physician services) and prescription drug claims (84). The Ontario Health Insurance Plan (OHIP) contains information on physician services, including diagnostic codes. The Discharge Abstract Database (DAD) and the National Ambulatory Care Reporting System (NACRS) contain all data on hospitalizations and emergency department visits, respectively. For each emergency visit or hospitalization, up to 25 possible diagnoses were registered according to the International Classification of Diseases system- tenth Revision (ICD-10). Of these entries, only one indicated the most reliable diagnosis or the main diagnosis. The administrative databases were linked using the unique and encrypted patient health insurance number and covered the period of April 24, 2012 to March 31, 2017.

\section{Ethics}

\section{Ethics approval}

was obtained for the study from the University of Alberta Health Research Ethics board (PRO 00083651) and Veritas Research Ethics Board in Ontario (16111-13:21:103-01-2017).

\section{Outcomes}

The study outcomes were 1) ED visit or hospitalization with a main diagnosis code for cannabis/cannabinoid poisoning (ICD-10 T40.7) and 2) ED visit or hospitalization with a main diagnosis code for mental or behavioral disorders due to the use of cannabis (ICD-10 F12).

\section{Potential Predictors}

Three groups of variables were assessed as potential predictors included sociodemographic variables, morbidities, and previous drug poisoning or substance use disorders. Sociodemographic variables included age at index date, sex, the nearest census-based neighborhood income quintile and the area of residence (rural versus urban). Selected morbidities based on previous studies $(7,9,10)$ included diabetes, congestive heart failure, chronic obstructive pulmonary disease, asthma, cancer, chronic kidney disease, liver disorders, musculoskeletal issues, pain, neurologic disorders, fatigue, metabolic disease. These variables were defined using ICD-9 codes (based on physician claim data) and ICD-10 codes (based on ambulatory care data and discharge abstract data). Prior drug poisonings or substance use disorders were defined as ED visit or hospitalization with codes specific to drug poisoning or mental and 
behavioural disorders. These included poisoning by: 1) narcotics and hallucinogens, 2) anesthetics and therapeutic gas, 3) hypno-sedative drugs, 4) other psychoactive drugs, and 5) alcohol as well as mental and behavioural disorders due to 1) psychoactive drug/substance, 2) alcohol, 3) Tobacco/nicotine, and 4) other mental and behavioural disorders (See Appendix A for more details).

\section{Statistical Analysis}

Descriptive statistics were used to assess the characteristics of the study sample (mean and standard deviation or median for continuous variables; numbers and proportions for categorical variables). Incidence rates per 10,000 person-years with $95 \%$ confidence intervals $(95 \% \mathrm{Cl})$ were calculated for the study outcomes.

To assess potential predictors of each of the study outcomes, we first assessed the correlation (Spearman correlation) between the potential predictors. Next, we conducted univariate Cox proportional hazard regression between each potential predictor and the outcomes, including the assessment of the models' assumptions. Next, variables that were associated at a p-value of 0.20 in univariate analysis were all included in a multivariate model, and then backward selection was used to retain the variables associated at a p-value $<0.10$.

For these analyses (i.e., predictors identification), we considered all primary and secondary diagnosis codes to define the outcomes as the number of events based on the primary diagnosis codes only was small. The analyses were performed using SAS version 9.4 (SAS Institute, Cary, NC, USA).

\section{Results}

From 29153 patients who received medical authorization to use cannabis 23091 satisfied the inclusion criteria (Fig. 1). The majority of them were aged 31 to 60 years and $53 \%$ were male (Table 1 ). The most prevalent morbidities were respectively musculoskeletal disorders (48.87\%), asthma (23.51\%), neurological disorders (21.82\%) and metabolic diseases (21.52\%) (Table 1). 
Table 1

Characteristics of the study population

\begin{tabular}{|c|c|c|}
\hline \multirow[t]{2}{*}{ Characteristics } & \multicolumn{2}{|c|}{ Total sample $(n=23091)$} \\
\hline & \multicolumn{2}{|l|}{$\mathrm{n}(\%)$} \\
\hline \multicolumn{3}{|l|}{ Age, years } \\
\hline$<21$ & 143 & $(0.62)$ \\
\hline $21-30$ & 2214 & $(9.59)$ \\
\hline $31-40$ & 4153 & $(17.99)$ \\
\hline $41-50$ & 4665 & $(20.20)$ \\
\hline $51-60$ & 6011 & $(26.03)$ \\
\hline $61-70$ & 3736 & $(16.18)$ \\
\hline $71-80$ & 1541 & $(6.67)$ \\
\hline$>80$ & 628 & $(2.72)$ \\
\hline \multicolumn{3}{|l|}{ Sex } \\
\hline Female & 10835 & $(46.92)$ \\
\hline Male & 12256 & $(53.08)$ \\
\hline \multicolumn{3}{|c|}{ Nearest census based neighbourhood income quintile } \\
\hline 1 & 5083 & $(22.01)$ \\
\hline 2 & 4766 & $(20.64)$ \\
\hline 3 & 4438 & $(19.22)$ \\
\hline 4 & 4619 & $(20.00)$ \\
\hline 5 & 4185 & $(18.12)$ \\
\hline Rural & 2317 & $(10.03)$ \\
\hline \multicolumn{3}{|l|}{ Morbidities } \\
\hline Asthma & 5428 & $(23.51)$ \\
\hline Cancer & 3215 & $(13.92)$ \\
\hline Congestive heart failure & 705 & $(3.05)$ \\
\hline Chronic obstructive pulmonary disease & 4059 & $(17.58)$ \\
\hline Diabetes & 3733 & $(16.17)$ \\
\hline Fatigue & 1282 & (5.55) \\
\hline
\end{tabular}




\begin{tabular}{|lcc|}
\hline Characteristics & \multicolumn{2}{l|}{ Total sample $(\mathbf{n}=\mathbf{2 3 0 9 1 )}$} \\
$\mathbf{n}(\%)$ & $(21.52)$ \\
\hline Metabolic disease & 4970 & $(48.88)$ \\
\hline Musculoskeletal disorders & 11288 & $(21.82)$ \\
\hline Neurological disorders & 5038 & $(8.71)$ \\
\hline Pain & 2012 & $(2.26)$ \\
\hline Chronic kidney disease & 522 & $(3.12)$ \\
\hline Liver disorders & 721 & $(0.42)$ \\
\hline Prior ED visit or hospitalization due to poisoning by: & 98 & $(0.39)$ \\
\hline narcotics and hallucinogens & 91 & $(0.25)$ \\
\hline Hypno-sedative drugs & 57 & $(1.10)$ \\
\hline Other psychoactive drugs & 177 & $(0.77)$ \\
\hline Prior ED visit or hospitalization for mental and behavioural disorder due to: & $(5.65)$ \\
\hline Psychoactive drugs/substances & 255 & \\
\hline Alcohol & 1304 & \\
\hline Other behavioural and mental disorders & & \\
\hline
\end{tabular}

During a median follow-up of 240 days and a total of 17353.8 person-years, 14 patients visited the ED or were hospitalized for cannabis poisoning, giving an incidence rate of 8.06 per 10,000 person-years patients (95\% Cl: 4.8-13.6) (Table 2).

Table 2

Incidence rates of emergency department (ED) visits or hospitalization for poisoning by cannabis and cannabis use disorders among patients with medical cannabis authorization

\begin{tabular}{|lll|}
\hline & $\begin{array}{l}\text { Outcome 1: Cannabis } \\
\text { poisoning }\end{array}$ & $\begin{array}{l}\text { Outcome 2: Cannabis-related mental and } \\
\text { behavioural disorders }\end{array}$ \\
\hline Total events & 14 & 26 \\
\hline Total person-years & 17353.8 & 17354.2 \\
\hline $\begin{array}{l}\text { Incidence rates per } \\
10,000\end{array}$ & $8.06(4.8-13.6)$ & $15.0(10.2-22.0)$ \\
$\begin{array}{l}\text { person-years (95\% } \\
\text { Cl) }\end{array}$ & & \\
\hline
\end{tabular}

A total of 26 patients visited the ED or were hospitalized for mental and behavioral disorders due to cannabis use for a total of 17354.2 person-years, which gave an incidence rate of 15.0 per 10,000 person- 
years (95\%Cl: 10.2-22.0) (Table 2).

Correlation between the assessed variables was small (highest correlation observed was between diabetes and metabolic disease: 0.53 ). By considering the secondary diagnosis codes in the definition of the outcomes for the predictor assessment only, the total events increased to 18 and 82 for cannabis poisoning and mental/behavioral disorders due to use of cannabis, respectively. There were very few observations for the co-variates poisoning by alcohol, by anesthetic and therapeutics gas and by tobacco/nicotine. Thus, results for these variables are not reported.

In univariate analyses, four variables were associated at a $p$-value $<0.10$ with poisoning by cannabis (Table 3). In the multivariate model with backward selection for these four variables, only two variables were retained: prior ED visit or hospitalization for poisoning by narcotics and hallucinogens ( $p$-value $=$ 0.0083 ) and sex ( $p$-value $=0.0793)$ (Table 4).

Table 3

Predictors of ED visit or hospitalization due to cannabis poisoning among patients with medical cannabis authorization in univariate analyses (with p-value $<0.10$ )

\begin{tabular}{|llll|}
\hline Variables & HR & $95 \% \mathrm{Cl}$ & P-value \\
\hline Sex (male versus female) & 0.412 & $0.154-1.099$ & 0.0764 \\
\hline ED visit or hospitalization for & & & \\
\hline poisoning by narcotics and hallucinogens & 15.75 & $2.089-118.776$ & 0.0075 \\
\hline other mental and behavioural disorders & 3.20 & $0.93-11.06$ & 0.0658 \\
\hline
\end{tabular}

Table 4

Predictors of ED visit or hospitalization due to cannabis poisoning among patients with medical cannabis authorization in multivariate analyses (with p-value $<0.10$ )

\begin{tabular}{|llll|}
\hline Variables & $\begin{array}{l}\text { Hazard } \\
\text { Ratio }\end{array}$ & $\begin{array}{l}95 \% \text { Confidence } \\
\text { interval }\end{array}$ & $\begin{array}{l}\text { p- } \\
\text { value }\end{array}$ \\
\hline $\begin{array}{l}\text { ED visit or hospitalization for poisoning by narcotics } \\
\text { and hallucinogens }\end{array}$ & 15.19 & $2.01-114.66$ & 0.0083 \\
\hline Sex (male versus female) & 0.42 & $0.16-1.11$ & 0.0793 \\
\hline
\end{tabular}

In univariate analyses 14 variables were associated with mental and behavioural disorders due to cannabis use (Table 5). In the multivariate model including these variables, two variables were associated at a $p$-value $<0.10$ (i.e., cancer and congestive heart failure) and seven variables were associated at a $p$ value $<0.05$ (Table 6). These latter variables were prior ED or hospitalization for poisoning by other psychoactive drugs, mental and behavioural disorder due to psychoactive drugs, and due to alcohol use, as well as other mental disorders, younger age, COPD, and diabetes. 
Table 5

Predictors of ED visit or hospitalization due to cannabis-related mental and behavioural disorders among patients with medical cannabis authorization in univariate analysis (with p-value $<0.10$ )

\begin{tabular}{|llll|}
\hline Predictors & HR & $95 \% \mathrm{Cl}$ & p-value \\
\hline Age (years) & & & \\
\hline $41-60$ versus $\leq 40$ & 0.400 & $0.247-0.647$ & 0.0002 \\
\hline$\geq 61$ versus $\leq 40$ & 0.357 & $0.188-0.677$ & 0.0016 \\
\hline Sex (male versus female) & 1.539 & $0.0 .979-2.421$ & 0.0619 \\
\hline Nearest census based neighbourhood income quintile & & & \\
\hline 2 versus 1 & 0.754 & $0.426-1.333$ & 0.3310 \\
\hline 3 versus 1 & 0.412 & $0.201-0.845$ & 0.0156 \\
\hline 4 versus 1 & 0.477 & $0.243-0.935$ & 0.0310 \\
\hline 5 versus 1 & 0.487 & $0.243-0.974$ & 0.0419 \\
\hline ED visit or hospitalization for & & & \\
\hline Poisoning by other psychoactive drugs (ICD-10 T43)* & 19.696 & $7.207-53.826$ & $<0.0001$ \\
\hline Mental and behavioural disorder due to psychoactive drugs & 11.701 & $5.855-23.383$ & $<0.0001$ \\
\hline Mental and behavioural disorder due to alcohol use & 13.643 & $6.572-28.321$ & $<0.0001$ \\
\hline Other mental and behavioural disorders & 6.76 & $4.20-10.87$ & $<0.0001$ \\
\hline Liver disorders & 2.207 & $0.894-5.452$ & 0.0862 \\
\hline Cancer & 0.290 & $0.092-0.920$ & 0.0356 \\
\hline Congestive heart failure & 2.861 & $1.246-6.569$ & 0.0132 \\
\hline Diabetes & 2.138 & $1.321-3.462$ & 0.0020 \\
\hline Fatigue & 1.868 & $0.901-3.873$ & 0.0933 \\
\hline Metabolic disease & 1.889 & $1.192-2.995$ & 0.0068 \\
\hline Pain & & $1.241-3.923$ & 0.0070 \\
\hline *include tricyclic and tetracyclic antidepressants, monoamine-oxidase-inhibitor antidepressants, \\
butyrophenone and thioxanthene neuroleptics, and other (see Appendix A for complete list) & \\
\hline
\end{tabular}


Table 6

Predictors of ED visit or hospitalization due to cannabis-related mental and behavioural disorders among patients with medical cannabis authorization in multivariate analysis (with $p$-value $<0.10$ )

\begin{tabular}{|c|c|c|c|}
\hline Variables & $\begin{array}{l}\text { Hazard } \\
\text { Ratio }\end{array}$ & $\begin{array}{l}95 \% \text { Confidence } \\
\text { Interval }\end{array}$ & $\begin{array}{l}\mathrm{P} \text { - } \\
\text { value }\end{array}$ \\
\hline \multicolumn{4}{|l|}{ ED visit or hospitalization for } \\
\hline $\begin{array}{l}\text { Poisoning by other psychoactive drugs (ICD-10 } \\
\text { T43)* }\end{array}$ & 5.18 & $1.83-14.63$ & 0.0019 \\
\hline $\begin{array}{l}\text { Mental and behavioural disorder due to } \\
\text { psychoactive drugs }\end{array}$ & 3.03 & $1.33-6.87$ & 0.0082 \\
\hline Mental and behavioural disorder due to alcohol use & 4.27 & $1.83-9.98$ & 0.008 \\
\hline Other mental and behavioural disorders & 3.88 & $2.32-6.51$ & $<.0001$ \\
\hline Age $41-60$ versus $\leq 40$ years & 0.33 & $0.19-0.58$ & 0.0001 \\
\hline Age $>60$ versus $\leq 40$ years & 0.29 & $0.14-0.63$ & 0.0016 \\
\hline Cancer & 0.37 & $0.12-1.21$ & 0.0999 \\
\hline Congestive heart failure & 2.35 & $0.92-5.99$ & 0.0730 \\
\hline COPD & 1.95 & $1.04-3.65$ & 0.0376 \\
\hline Diabetes & 2.63 & $1.54-4.47$ & 0.0004 \\
\hline \multicolumn{4}{|c|}{$\begin{array}{l}\text { *include tricyclic and tetracyclic antidepressants, monoamine-oxidase-inhibitor antidepressants, } \\
\text { butyrophenone and thioxanthene neuroleptics, and other (see Appendix A for complete list) }\end{array}$} \\
\hline
\end{tabular}

\section{Discussion}

This study shows that rates of ED visits or hospitalization for poisoning by cannabis and cannabisrelated mental and behavioral disorders was low among patients with medical cannabis authorization. Two predictors were identified for cannabis poisoning (i.e., prior poisoning by narcotics or hallucinogens and sex). For cannabis-related mental and behavioral ED visits and hospitalization, seven predictors were identified, including prior poisoning by other psychoactive drugs (antidepressants and other), mental and behavioral disorder due to psychoactive drugs or alcohol, other mental and behavioral disorders, younger age, diabetes, COPD.

Our observation of low rates of ED visits and hospitalization for cannabis poisoning and cannabis use disorders among this large cohort of medical cannabis users helps address concerns regarding increasing use of medical cannabis. However, for CUD, the low rate could also be explained by the relative short length of follow-up which might not allow sufficient time to observe onset and diagnosis among 
those patients. Studies with people who use medical cannabis that have a longer follow-up period are needed to fully address this question. Very few studies have assessed the rates of cannabis poisoning and cannabis use disorders among medical cannabis patients(18). Available data are mostly based on recreational cannabis users or a mix of both recreational and medical cannabis users $(11,19)$ with no possibility to distinguish rates for medical cannabis users only.

Regarding the characteristics of patients that might have an increased risk of cannabis poisoning or cannabis use disorders, our results suggest that patients with history of drug poisoning or substance use disorder, and more generally patient with mental and behavioral disorders, should be regarded as patients with higher susceptibility. Consistent with previous data $(9,10)$, our results suggest that these conditions should be systematically assessed among patients seeking medical. This suggestion aligns with the recommendation of the $\mathrm{CCSMH}(20)$, calling for proactive vigilance among clinicians to detect a history of CUD in patients seeking medical cannabis. The observation that younger medical cannabis use patients are at higher risk of cannabis use disorders is also observed in a previous US nationwide sample that showed that hospitalizations for cannabis use disorders were more prevalent among younger patients (9). In this US study, diabetes, COPD and CHF were also prevalent conditions among patients who were hospitalized for cannabis use disorders(9). However, why patients with diabetes and COPD using cannabis are at higher risk of cannabis use disorders, as we observed in our data, remains unclear.

The primary strength of this study is that it is currently the largest (to our knowledge) Canadian population-based cohort study of medical cannabis authorized patients. It is also the only study that specifically studies the incidences rates and predictive factors of hospitalization from CUD/cannabis poisoning in an adult population in Canada. However, our study is not without limitations. Firstly, our study was unable to capture CUD or cannabis intoxication cases that did not result in hospitalization/ED visit. As this was an observational study, potential spectrum bias is a concern since our cohort is based on patients who individually sought authorization for medical cannabis. Secondly, this population may not be representative of all individuals who are using cannabis for medical purposes but may have obtained non-medical cannabis via other (legal or illegal) avenues. Notably, our study cannot ensure cannabis products were consumed as authorized or if patients elected to use alternative agents than what was authorized. Thirdly, information on other prescribed medications was only available for a subset of the study population as only patients $w$ or concomitant use of other substances, which may have varied between the two populations.

\section{Conclusion}

In all, this cohort study suggests that incidence of cannabis poisoning or cannabis use disorders was low among patients who were authorized by healthcare providers to use cannabis for therapeutic reasons. Important predictors were identified and should be considered during cannabis prescription for a better benefit-risk assessment. More particularly, the results suggest that patients with prior drug use disorders or mental and behavioral disorders should be regarded as at higher risk of cannabis use disorders. 


\section{Abbreviations}

COPD

chronic obstructive pulmonary disorder

$\mathrm{CHF}$

congestive heart failure

CUD

cannabis use disorder

DAD

Discharge Abstract Database

ED

emergency department

ICD-19

International Classification of Diseases System - tenth revision

ICES

Institute for Clinical Evaluative Sciences

NACRS

National Ambulatory Care Reporting System

OHIP

Ontario Health Insurance Plan

\section{Declarations}

\section{Ethics approval and consent to participate}

All methods in this study were performed in accordance with the relevant guidelines and regulations as outlined in the Declaration of Helsinki and has been approved by an appropriate ethics committee. Ethics approval was obtained for the study from the University of Alberta Health Research Ethics board (PRO 00083651) and Veritas Research Ethics Board in Ontario (16111-13:21:103-01-2017). Patients and the public were not involved in the design, conduct and reporting of this research project as it was not applicable to this project.

\section{Consent for publication}

This study made use of de-identified data from the Ontario administrative data, which is managed by ICES with support from its funders and partners: Canada's Strategy for Patient-Oriented Research (SPOR), the Ontario SPOR Support Unit, the Canadian Institutes of Health Research and the Government of Ontario. The opinions, results and conclusions reported are those of the authors. No endorsement by ICES or any of its funders or partners is intended or should be inferred. Parts of this material are based on data and information compiled and provided by Canadian Institute of Health Information (ClHI). However, the analyses, conclusions, opinions and statements expressed herein are only those of the authors. 


\section{Availability of data and materials}

The dissemination of data results to study participants and or patient organizations in this research project is not possible/applicable as the data are de-identified. Being administrative health data, the data cannot be shared publicly. However, requests for the data can be sent to ICES. No special access privileges were granted to the authors.

\section{Competing interests}

JRBD was a former member on the board of directors of Aurora Cannabis Inc., which is a for-profit, company licensed for the cultivation and sale of medical cannabis. In the past, JGH has worked as a paid advisor and speaker for Canadian Cannabis Clinics, but currently has no ties with the CCCs. JRBD has a financial interest in Aurora Cannabis Inc. DTE and JRBD hold a Mitacs Grant with Aurora as a partner. Mitacs is a national, not-for-profit organization that works with universities, private companies, and both federal and provincial governments, to build partnerships and administer research funding that supports industrial and social innovation in Canada. DTE and AZ do not have any past or present financial interest in the companies involved. $\mathrm{CL}, \mathrm{JM}$ and $\mathrm{EH}$ have no conflicts of interest to declare. Moreover, the above mentioned entities, research funders and companies listed were not involved in any aspect of the design or write-up of the study and all analysis was performed independent from the funders and companies.

\section{Competing interests}

JRBD is a former member on the board of directors of Aurora Cannabis Inc., which is a for-profit, company licensed for the cultivation and sale of medical cannabis. In the past, JGH has worked as a paid advisor and speaker for Canadian Cannabis Clinics, but currently has no ties with the CCCs. JRBD has a financial interest in Aurora Cannabis Inc. DTE and JRBD hold a Mitacs Grant with Aurora as a partner. Mitacs is a national, not-for-profit organization that works with universities, private companies, and both federal and provincial governments, to build partnerships and administer research funding that supports industrial and social innovation in Canada. DTE and AZ do not have any past or present financial interest in the companies involved. $\mathrm{CL}, \mathrm{JM}$ and $\mathrm{EH}$ have no conflicts of interest to declare. Moreover, the above mentioned entities, research funders and companies listed were not involved in any aspect of the design or write-up of the study and all analysis was performed independent from the funders and companies.

\section{Funding}

This study was funded by a Canadian Institutes of Health research Project grant (CIHR PS 159668) to DTE, JGH, EH, and JRBD. The funder has no role in the design of the study, analysis and interpretation of the data, and the decision to publish.

\section{Authors' contributions}

DTE, AZ, JRBD, JGH, EH designed the study and DTE and JRBD acquired the data. AZ analyzed the data. AZ, CL and JM drafted the manuscript. All other authors revised it critically for important intellectual 
content and approved the final version to be published. All authors are accountable for the work and integrity of the work. The corresponding author and guarantor accept full responsibility of the work and/or conduct of the study, had access to the data and controlled the decision to publish. AZ attests that all listed authors meet authorship criteria and that no others meeting the criteria have been omitted.

AZ affirms that the manuscript is an honest, accurate, and transparent account of the study being reported; that no important aspects of the study have been omitted; and that any discrepancies from the study as planned (and if relevant) have been explained.

\section{References}

1. Sahlem GL, Tomko RL, Sherman BJ, Gray KM, McRae-Clark AL. Impact of cannabis legalization on treatment and research priorities for cannabis use disorder. Int Rev Psychiatry. 2018;30(3):216-25.

2. Wilkinson ST, Yarnell S, Radhakrishnan R, Ball SA, D'Souza DC. Marijuana Legalization: Impact on Physicians and Public Health. Annu Rev Med. 2016;67:453-66.

3. Budney AJ, Sofis MJ, Borodovsky JT. An update on cannabis use disorder with comment on the impact of policy related to therapeutic and recreational cannabis use. Eur Arch Psychiatry Clin Neurosci. 2019;269(1):73-86.

4. Patel JM, R. Cannabis Use Disorder. Internet: StatPearls; 20202020 January.

5. Loflin MJE, Kiluk BD, Huestis MA, Aklin WM, Budney AJ, Carroll KM, et al. The state of clinical outcome assessments for cannabis use disorder clinical trials: A review and research agenda. Drug Alcohol Depend. 2020;212:107993.

6. Ksir C, Hart CL. Cannabis and Psychosis: a Critical Overview of the Relationship. Curr Psychiatry Rep. 2016;18(2):12.

7. Salas-Wright CP, Carbone JT, Holzer KJ, Vaughn MG. Prevalence and correlates of cannabis poisoning diagnosis in a national emergency department sample. Drug Alcohol Depend. 2019;204:107564.

8. Yeung MEM, Weaver CG, Janz K, Haines-Saah R, Lang E. Clearing the air: A study of cannabis-related presentations to urban Alberta emergency departments following legalization. CJEM. 2020:1-8.

9. Charilaou P, Agnihotri K, Garcia P, Badheka A, Frenia D, Yegneswaran B. Trends of Cannabis Use Disorder in the Inpatient: 2002 to 2011. Am J Med. 2017;130(6):678-87 e7.

10. Ware MA, Martel MO, Jovey R, Lynch ME, Singer J. A prospective observational study of problematic oral cannabinoid use. Psychopharmacology (Berl). 2018;235(2):409-17.

11. Pinto JV, Medeiros LS, Santana da Rosa G, Santana de Oliveira CE, Crippa JAS, Passos IC, et al. The prevalence and clinical correlates of cannabis use and cannabis use disorder among patients with bipolar disorder: A systematic review with meta-analysis and meta-regression. Neurosci Biobehav Rev. 2019;101:78-84.

12. Lucatch AM, Coles AS, Hill KP, George TP. Cannabis and Mood Disorders. Curr Addict Rep. 2018;5(3):336-45. 
13. Patel RS, Mekala HM, Tankersley WE. Cannabis Use Disorder and Epilepsy: A Cross-National Analysis of 657072 Hospitalized Patients. Am J Addict. 2019;28(5):353-60.

14. Dervaux A. [Cannabis use disorders and psychiatric comorbidities]. Rev Prat. 2018;68(6):670-4.

15. Brezing CA, Levin FR. The Current State of Pharmacological Treatments for Cannabis Use Disorder and Withdrawal. Neuropsychopharmacology. 2018;43(1):173-94.

16. Eurich D, Lee C, Zongo A, Minhas-Sandhu JK, Hanlon JG, Hyshka E, et al. Cohort study of medical cannabis authorisation and healthcare utilisation in 2014-2017 in Ontario, Canada. J Epidemiol Community Health. 2020;74(3):299-304.

17. Eurich D, Hanlon JG., Boisvenue, JJ., Meng, H., \& Dyck, J.R.B. A Description of Medical Cannabis Use in Ontario, Canada. Cannabis and Cannabinoid Research. 2019;4(2):131-5.

18. Gage SH, Hickman M, Zammit S. Association Between Cannabis and Psychosis: Epidemiologic Evidence. Biol Psychiatry. 2016;79(7):549-56.

19. Blanco C, Hasin DS, Wall MM, Florez-Salamanca L, Hoertel N, Wang S, et al. Cannabis Use and Risk of Psychiatric Disorders: Prospective Evidence From a US National Longitudinal Study. JAMA Psychiatry. 2016;73(4):388-95.

20. Bertram JR, Porath A, Seitz D, Kalant H, Krishnamoorthy A, Nickerson J, et al. Canadian Guidelines on Cannabis Use Disorder Among Older Adults. Can Geriatr J. 2020;23(1):135-42.

\section{Figures}

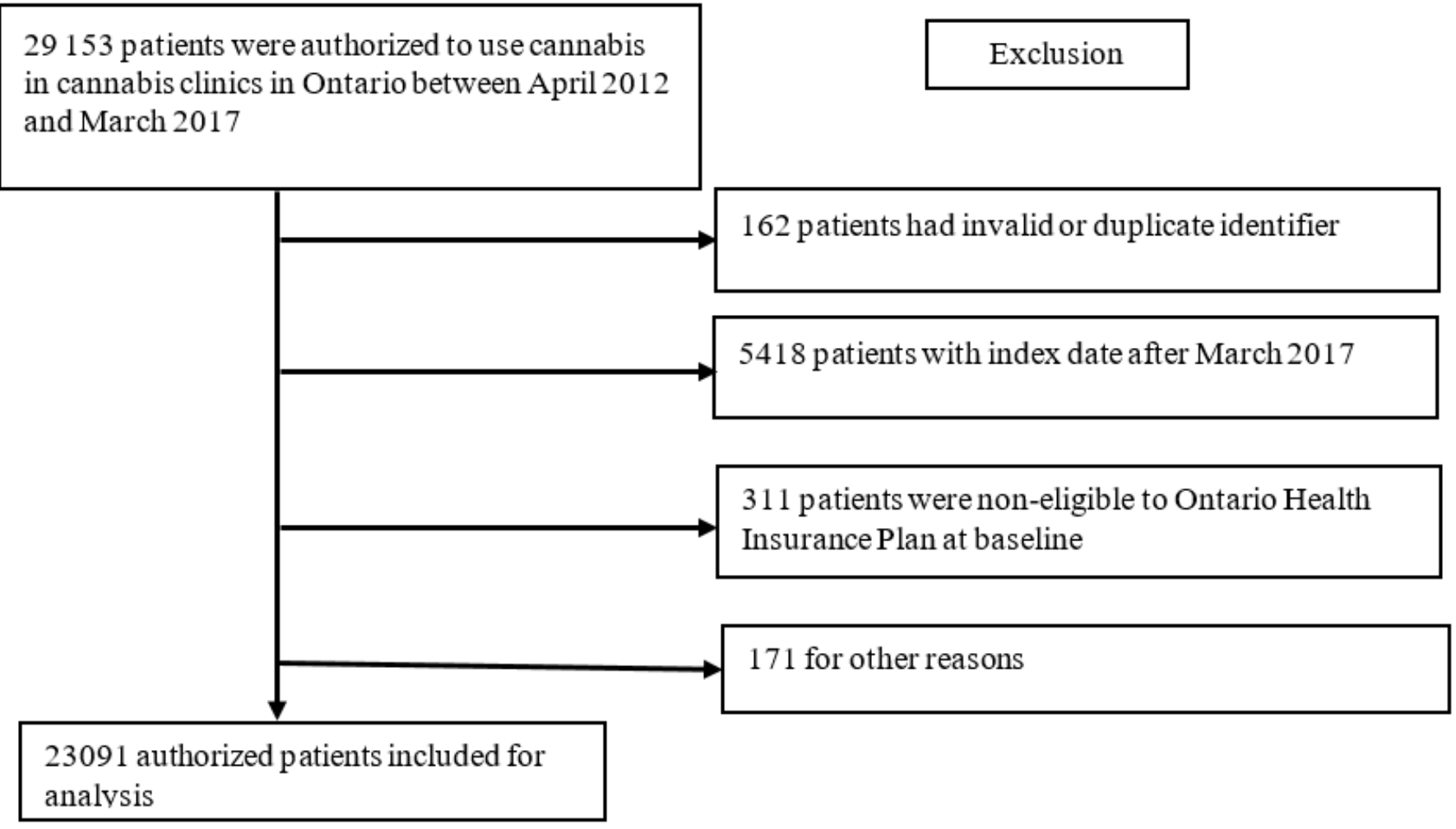


Selection of study population

\section{Supplementary Files}

This is a list of supplementary files associated with this preprint. Click to download.

- Appendix.docx 